\section{Describing Function Properties of a Magnetic Pulsewidth Modulator}

\author{
R.D. MIDDLEBROOK, Senior Member, IEEE \\ California Institute of Technology \\ Pasadena, Calif. 91109
}

\begin{abstract}
An analysis is presented for the transfer functions of a particular pulsewidth modulator and power-stage subsystem that has been widely used in practical switching-mode dc regulator systems. The switch and filter are in a "buck" configuration, and the switch is driven by a constant-frequency, variable duty-ratio, push-pull magnetic modulator employing square-loop cores. The two transfer functions considered are that with modulator control signal as input and that with line voltage as input. For ac signals, the corresponding describing functions (DF) are derived. It is shown that currentsource drive to the modulator extends the control DF frequency response over that with voltage drive, and that complete cancellation of the effects of line variations can be obtained at dc, but not for ac. Experimental confirmation of the analytical results for the control DF are presented.
\end{abstract}

\section{Introduction}

In switching-mode dc regulators and amplifiers, a control signal modulates the duty ratio of a switch associated with a line voltage and a low-pass filter. This subsystem of modulator and power stage is usually part of an overall feedback loop, as shown schematically in Fig. 1, whose regulation and stability depend upon two properties of the subsystem: the control signal to output voltage transfer function and the line voltage to output voltage transfer function. The dc transfer functions are easily understood and analyzed, but analysis of the ac transfer functions presents considerable difficulties, since the subsystem effectively contains an analog-to-digital and a digital-to-analog converter, and is thus inherently nonlinear. For many practical systems, the problem of absence of quantitative understanding of the ac transfer functions is merely avoided by introduction of a sufficiently low-frequency dominant pole in the overall feedback loop to ensure stability. The result is poorer system performance than might otherwise be obtained.

This paper presents an analysis for the transfer functions of a particular modulator and power-stage subsystem. However, before introduction of the particular subsystem, a more detailed discussion of the nature of the generalized subsystem of Fig. 1 will be given, in order to establish the specific definitions of the analysis goals.

If the line voltage $v_{\ell}(t)$ has a constant dc value $V_{\ell}$ and the control signal is a constant dc voltage $V_{c}$, the modulator delivers a digital switch drive that turns the switch on and off with a constant duty ratio $D$ and a constant repetition, or switching, frequency $f_{s}$. The output voltage $v_{0}(t)$ contains a constant dc component $V_{0}$, and also harmonics of the switching frequency $f_{s}$.

If, again, the line voltage is constant at $V_{\ell}$, but the control signal consists of a dc voltage $V_{c}$ plus a sinusoidal component $\hat{v}_{c}$ at frequency $f$, where $\hat{v}_{c}$ is expressed in phasor form, the duty ratio, and perhaps also the switching frequency, change at the frequency $f$, and the resulting output voltage contains, in addition to the dc component $V_{0}$ and harmonics of $f_{s}$, a phasor component $\hat{v}_{0}$ at the control frequency $f$ and components at higher harmonics of $f$ as well as sidebands at sum and difference frequencies of the harmonics of $f_{s}$ and $f$. A statement of the complete control signal to output voltage transfer function is therefore very complicated, and any attempt to determine the stability of the overall feedback loop is even more so. What one would like is to ignore all components of the output voltage other than those at dc and the control frequency; the remaining components, at least for control frequencies much lower than the switching frequency, are, in fact, purposely made small by inclusion of the low-pass filter. The relation between a sinusoidal input signal and the resulting output voltage component at the same frequency $\omega=2 \pi f$, where both are expressed in phasor form, is known as the describing function; so the control signal to output voltage describing function $F_{c}$ is defined as

$$
F_{c}(j \omega)=\frac{\hat{v}_{0}}{\hat{v}_{c}}
$$

Presented at the Third IEEE Power Processing and Electronic Specialists Conference, Atlantic City, N.J., May 1972.

Manuscript received October 30, 1972. 


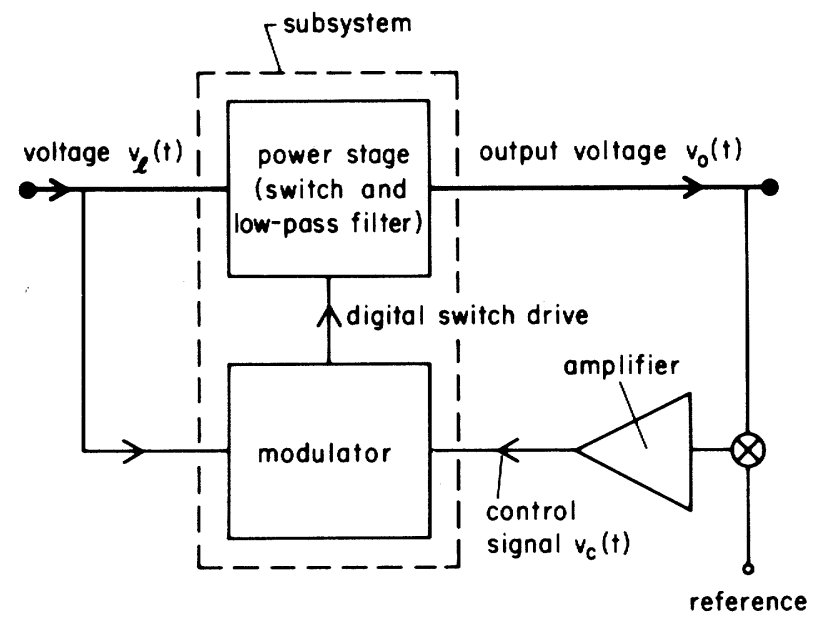

Fig. 1. Block diagram of typical closed-loop dc regulator, including a subsystem containing a modulator and switched power stage.

under conditions of constant line voltage $v_{\ell}(t)=V_{\ell}$.

A similar discussion may be made for the condition in which the control signal is constant, but the line voltage consists of a dc voltage $V_{\ell}$ plus a sinusoidal component $\hat{v}_{\ell}$ in phasor form at frequency $\omega=2 \pi f$. Output voltage components at frequencies other than $f$ are ignored, and the line voltage to output voltage describing function is defined as

$$
F_{\ell}(j \omega)=\frac{\hat{v}_{0}}{\hat{v}_{\ell}}
$$

under conditions of constant control signal $v_{c}(t)=V_{c}$.

It may be noted that neither describing function may be linear, in that its value may depend upon the amplitude of the relevant sinusoidal input signal. It is therefore convenient to define the linearized describing function as the limit of the describing function as the amplitude of the input signal becomes vanishingly small. The usefulness of the linearized describing function is that the actual nonlinear subsystem of Fig. 1 can be approximately modeled by a "black box" characterized by linear transfer functions. As a result, the large body of linear system theory can be invoked to investigate the performance and stability of the complete regulator or amplifier. It must be remembered, however, that because of the approximations implicit in the linearized describing function representation, a stability criterion, as obtained, for example, from a Nyquist plot, constitutes a necessary but perhaps not sufficient condition for stability.

There are many ways in which the power stage and modulator of Fig. 1 can be implemented in practical systems. For example, the power stage may be implemented by a switch and LC filter in the familiar buck, boost, or buck-boost configuration. The modulator, of necessity, samples the control signal to produce the digital switch drive; the sampling may be uniform, natural, or any of various integrating types, and the resulting digital switch drive may

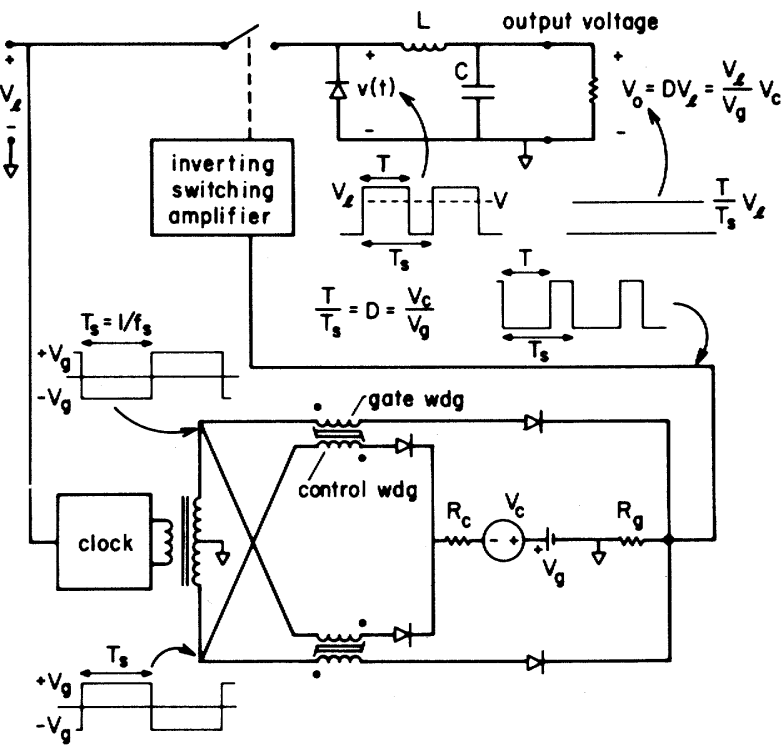

Fig. 2. A specific subsystem consisting of a power switch and filter in buck configuration and a constant-frequency, variable duty-ratio, push-pull, magnetic modulator employing square-loop cores.

be constant on-time, constant off-time, or constant onplus-off-time (constant, or clocked, frequency).

The purpose of this paper is to present analytical expressions for the linearized control signal to output voltage and line voltage to output voltage describing functions for a particular modulator and power-stage subsystem that has been widely used in practical switching-mode $\mathrm{dc}$ regulator systems.

\section{Qualitative Operation of a Magnetic Modulator and Power Stage}

The particular implementation of the modulator and power-stage subsystem to be considered is shown in Fig. 2 , in which the waveforms are those for steady-state, or $\mathrm{dc}$, operation, where the line voltage is constant at $V_{\ell}$ and the control signal is a constant voltage $V_{c}$. For analysis purposes, all diodes and the power switch are assumed ideal. The switch and filter are in a buck configuration, and the switch is driven by a constant-frequency, variable duty-ratio, push-pull magnetic modulator employing square-loop cores. The control windings are shown driven by a Thevenin equivalent source voltage $V_{c}$ with resistance $R_{c}$, and the gate windings are driven from a square-wave clock oscillator of frequency $f_{s} / 2$. The principle of operation is well known, and need be only briefly summarized. The volt-seconds stored in one core by the control winding during a period $T_{s}=1 / f_{s}$ are recovered through the gate winding during an interval $T$ of the following period, during which interval the gate winding supports the clock voltage $V_{g}$, there is no drive to the inverting switching amplifier, and the power switch is closed. The interval $T$ is determined by the voltseconds stored and by the gate voltage $V_{g}$. At the end of 


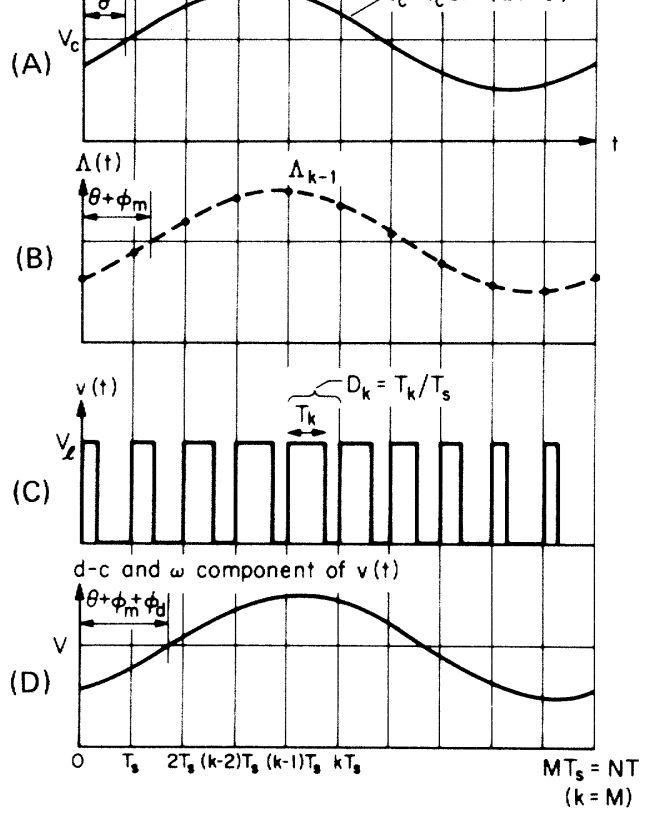

Fig. 3. Waveforms in the subsystem of Fig. 2 for dc plus sinusoidal control signal.

the interval $T$ the core saturates and the voltage $V_{g}$ appears across the resistance $R_{g}$ and is then applied to the inverting switching amplifier so that the power switch is opened for the remainder of the period $T_{s}$. The diodes and the square-wave clock voltage ensure that the cores perform these functions alternately and without interaction between the control and gate windings. The battery $V_{g}$ is included as an analytical convenience to cancel the steering clock voltage, so that, for zero source resistance $R_{c}$, the voltage applied to the active control winding is $V_{c}$. Because of the push-pull nature of the modulator, the power switch repetition rate (the switching frequency $f_{s}$ ) is twice the frequency of the square-wave clock oscillator.

The subsystem may be classed as a buck power stage driven by a constant-frequency, or clocked, uniformly sampling integrating modulator. The control winding voltage is integrated over a period $T_{s}$, and is sampled uniformly at intervals $T_{S}$ to determine the duty ratio $D=T / T_{S}$ of the power switch drive for the following period $T_{s}$. Quantitatively, again for steady-state or dc conditions and for zero source resistance $R_{c}$, the volt-seconds $\Lambda_{0}$ stored by the control winding voltage $V_{c}$ over a period $T_{s}$ are $\Lambda_{0}=V_{c} T_{s}$, and the same volt-seconds are then recovered under the gate voltage $V_{g}$ to determine the following interval $T$, so that

$$
D=\frac{T}{T_{s}}=\frac{V_{c}}{V_{g}} .
$$

When the switch is closed, the voltage $v(t)$ at the filter input is equal to the line voltage $V_{\ell}$; and if the conditions of operation of the power stage are constrained (as is usual) so that the inductor current never falls to zero, the diode clamps the voltage at the input of the filter to zero when the switch is open. It follows that the dc component $V$ of the switched voltage $v(t)$ at the filter input is simply equal to the time average of $V_{\ell}$ and zero, namely

$$
V=D V_{\ell}
$$

Since the dc component of $v(t)$ is unaffected by the filter, the final result is

$$
\begin{aligned}
V_{0} & =V=D V_{\ell} \\
& =\frac{V_{\ell}}{V_{g}} V_{c} .
\end{aligned}
$$

Equation (6) represents the basic dc operation of the modulator and power-stage subsystem, and contains both the control signal to output and line voltage to output transfer functions. For constant line voltage, the dc output voltage is proportional to the dc control voltage, so the control to output transfer function is a constant. If may be seen that the possibility exists of making the line voltage to output transfer function zero. All that is required is to make $V_{g}$ proportional to $V_{\ell}$, which is easily achieved, at least to first-order approximation, by making the amplitude of the clock oscillator output proportional to the line voltage.

Equation (6) also contains the two transfer functions with respect to incremental changes of sufficiently low frequency, that is, the two describing functions already introduced. Thus, $F_{c}=V_{\ell} / V_{g}$ and $F_{\ell}=0$ for sufficiently low-frequency variations. However, because of the integration function of the modulator and the delay function inherent in the digital switch drive, at higher frequencies the output voltage ceases to follow the control signal, and also, cancellation of the line voltage variations ceases to be complete. The principal objective of the following analysis is to derive the two linearized describing functions $F_{c}(j \omega)$ and $F_{l}(j \omega)$ as functions of frequency, and to determine how these functions depend upon the resistance $R_{c}$ of the control signal source and on appropriate parameters of the magnetic cores.

The problem of determination of the control signal to output voltage describing function can be formulated as follows. Let the line voltage be constant at $V_{\ell}$, and let the control signal be a dc plus a sinusoidal ac voltage given by

$$
v_{c}(t)=V_{c}+v_{c} \sin (\omega t-\theta)
$$

where $t=0$ is taken at the start of one of the switching periods $T_{s}$. This waveform is shown in Fig. 3(A). The duty ratio $D_{k}$ for the $k$ th period $T_{s}$ is determined by the stored volt-seconds $\Lambda_{k-1}$ at the beginning of that period. It will be shown that the "samples" $\Lambda_{k-1}$ are points on a sine wave given by

$$
\Lambda_{k-1}=\Lambda_{0}+\lambda \sin \left[(k-1)\left(\frac{2 \pi \omega}{\omega_{s}}\right)-\theta-\phi_{m}\right] .
$$

This sine wave and the uniform samples are shown in Fig. 3(B). The waveform $v(t)$ at the input to the filter is as shown in Fig. 3(C); it is a square wave of constant ampli- 
tude $V_{\ell}$ and constant repetition rate $f_{s}$, but of varying duty ratio. The interval $T_{k}$ during which the power switch is closed is determined by the time taken to recover the stored volt-seconds $\Lambda_{k-1}$ under the constant gate voltage $V_{g}$, so $T_{k}=\Lambda_{k-1} / V_{g}$ and the $k$ th duty ratio $D_{k}$ is

$$
D_{k}=\frac{\Lambda_{k-1}}{V_{g} T_{s}} .
$$

Shown in Fig. 3(D) are the dc component $V$ and the component at the control frequency $\omega$ of the waveform $v(t)$. It will be shown that, with neglect of all other components, the voltage at the filter input is given by

$$
v(t)=V+v \sin \left(\omega t-\theta-\phi_{m}-\phi_{d}\right) .
$$

The component of the output voltage at the control frequency is merely the filter input voltage at that frequency modified by the filter characteristic, so that the control signal to output voltage describing function can be separated into two factors:

$$
F_{c}(j \omega)=F_{c}^{\prime}(j \omega) F(j \omega)
$$

where $F(j \omega)$ is the linear filter characteristic and $F_{c}^{\prime}(j \omega)$ is the describing function from the control signal to the filter input voltage. Henceforth, this describing function will be referred to as the control DF. From (7) and (10), the control DF $F_{c}^{\prime}(j \omega)$ is given by

$$
F_{c}^{\prime}(j \omega)=\frac{v}{v_{c}} \exp \left[-j\left(\phi_{m}+\phi_{d}\right)\right]
$$

It remains to determine the magnitude $v / v_{c}$ and the phase lag $\phi_{m}+\phi_{d}$ of the control DF as functions of frequency and the various circuit parameters.

The problem of determination of the line voltage to output voltage describing function is formulated in a similar manner. Let the control signal be a constant voltage $V_{c}$, and let the line voltage be a dc plus a sinusoidal ac voltage given by

$$
v_{\ell}(t)=V_{\ell}+v_{\ell} \sin (\omega t-\theta)
$$

where $t=0$ is again taken at the start of one of the switchperiods $T_{s}$. This waveform is shown in Fig. 4(A). Also, let the gate voltage be proportional to the line voltage by a factor $B$, so that

$$
v_{g}(t)=B v_{\ell}(t)=B\left[V_{\ell}+v_{\ell} \sin (\omega t-\theta)\right],
$$

and the desired complete cancellation in the output of dc line variations is achieved. Since the control signal is constant, the stored volt-seconds at the sampling instants are constants at a value $\Lambda_{0}$, as shown in Fig. 4(B). The waveform $v(t)$ at the input to the filter is shown in Fig. $4(\mathrm{C})$; when the power switch is closed, $v(t)$ is equal to the line voltage $v_{\ell}(t)$. If the duty cycle were constant at a value $D, v(t)$ would contain a component of amplitude $D v_{\ell}$ at the frequency $\omega$. However, because the gate voltage is proportional to the line voltage, the constant stored volt-seconds
(A)

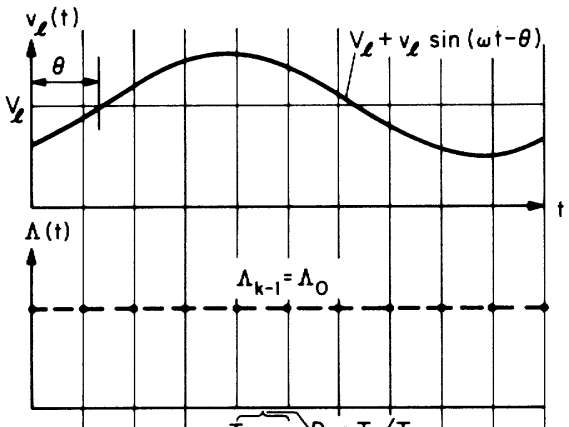

(C)

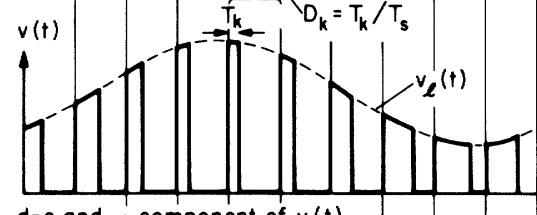

(D)

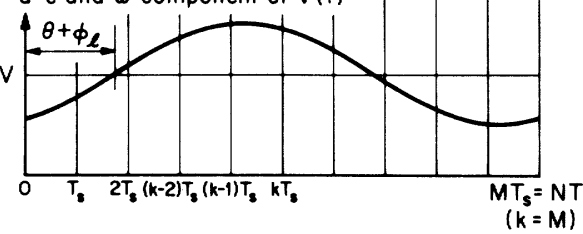

Fig. 4. Waveforms in the subsystem of Fig. 2 for dc plus sinusoidal line voltage.

$\Lambda_{0}$ are recovered over an interval $T_{k}$ that varies with $v_{g}(t)$ and, hence, with $v_{\ell}(t)$, so that $D_{k}$ is not constant but varies in such a way that the component of $v(t)$ at the frequency $\omega$ has an amplitude smaller than $D v_{\ell}$. It will be shown that, with neglect of all other sinusoidal components, the voltage at the filter input is given by

$$
v(t)=V+v \sin \left(\omega t-\phi_{\ell}\right)
$$

as shown in Fig. 4(D). Again, the line voltage to output voltage describing function can be separated into two factors:

$$
F_{\ell}(j \omega)=F_{\ell}^{\prime}(j \omega) F(j \omega)
$$

where $F_{\ell}^{\prime}(j \omega)$ is the describing function from the line voltage to the filter input voltage. Henceforth, this describing function will be referred to as the line DF. From (13) and (15), the line $\mathrm{DF} F_{\ell}^{\prime}(j \omega)$ is given by

$$
F_{\ell}^{\prime}(j \omega)=\frac{v}{v_{\ell}} \exp \left(-j \phi_{\ell}\right),
$$

and it remains to determine the magnitude $v / v_{\ell}$ and the phase lag $\phi_{\ell}$ of the line DF as functions of frequency and the various circuit parameters.

The procedure outlined above for determination of the control and line DF's will now be traced in detail.

\section{The Control Describing Function}

To obtain an analytical expression for the control DF, the first step is to find the stored volt-seconds $\Lambda_{k-1}$ at the 
sampling instants $t=(k-1) T_{s}$. The case of zero source resistance $R_{c}=0$ will be treated first, since it is particularly simple and affords a useful reference for the general case.

If $R_{c}=0$, the control-signal voltage $v_{c}(t)=V_{c}+v_{c}$ sin $(\omega t-\theta)$ is applied directly across the control winding. The core starts at, say, negative saturation, and the volt-seconds $\Lambda_{k-1}$ stored during the period $(k-2) T_{s}<t<(k-1) T_{s}$ are given by

$$
\Lambda_{k-1}=\int_{(k-2) T_{s}}^{(k-1) T_{s}}\left[V_{c}+v_{c} \sin (\omega t-\theta)\right] d t .
$$

The result is

$$
\begin{aligned}
\Lambda_{k-1}= & V_{c} T_{s}+v_{c} T_{s} \frac{\sin \left(\pi \omega / \omega_{s}\right)}{\left(\pi \omega / \omega_{s}\right)} \\
& \cdot \sin \left[(k-1)\left(\frac{2 \pi \omega}{\omega_{s}}\right)-\theta-\left(\frac{\pi \omega}{\omega_{s}}\right)\right]
\end{aligned}
$$

which is of the form given in (8), in which

$$
\begin{aligned}
\Lambda_{0} & =V_{c} T_{s} \\
\lambda & =v_{c} T_{s} \frac{\sin \left(\pi \omega / \omega_{s}\right)}{\left(\pi \omega / \omega_{s}\right)} \\
\phi_{m} & =\frac{\pi \omega}{\omega_{s}} .
\end{aligned}
$$

It may be observed that the properties of the cores have not entered into this result, except that the $B-H$ loop has been assumed sufficiently square that the qualitative operation is maintained.

In the general case, where the control source resistance is not zero, the voltage applied across the control winding is less than the control voltage by the drop in $R_{c}$. Since this drop is dependent upon the current in the control winding, the properties of the core that relate current to voltage must now enter into the result. The model used to represent the properties of the cores is shown in Fig. 5. This is a $B-H$ square-loop characteristic transformed to a $\Lambda-I$ or volt-second-magnetizing current characteristic, as seen by either the control or gate winding, which, for simplicity, are taken to have equal turns. Two principal second-order features are included in the model. First, the zero-flux magnetizing current $I_{m}$ increases over its static value $I_{0}$ in proportion to the winding voltage $d \Lambda / d t$, or

$$
I_{m}-I_{0}=\frac{1}{R} \frac{d \Lambda}{d t}
$$

where $R$ has the nature of a resistance. This assumed linear relation between excess magnetizing current and voltage is an approximate representation of the well-known "loop widening effect." Second, the unsaturated part of the core characteristic is not vertical, but represents a noninfinite inductance $L$. The two core-dependent quantities that affect the modulator properties are $R$ and $\tau \equiv L / R$, where the resistance $R$ is a function of the core material, geometry,

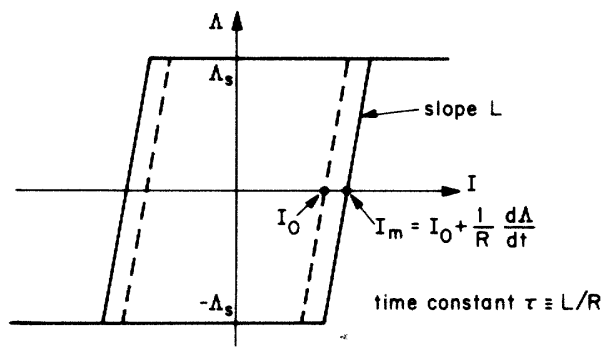

Fig. 5. Core model, including two second-order parameters, the loop-widening resistance $R$ and the noninfinite unsaturated inductance $L$.

and number of turns, and the time constant $\tau$ is a property of the core material only.

During any period $T_{s}$, the voltage across the control winding is $d \Lambda / d t$, and so the current through $R_{c}$ is $I=\left[v_{c}(t)-d \Lambda / d t\right] / R_{c}$. This is also the total magnetizing current of the core $I=I_{m}+\Lambda / L$. Hence,

$$
v_{c}(t)-\frac{d \Lambda}{d t}=R_{c}\left(I_{m}+\frac{\Lambda}{L}\right) .
$$

After substitution for $v_{c}(t)$ and $I_{m}$, the differential equation for $\Lambda$ is

$$
\begin{aligned}
\frac{d \Lambda}{d t}+r \frac{\Lambda}{\tau}= & (1-r) V_{c}-r R I_{0} \\
& +(1-r) v_{c} \sin (\omega t-\theta)
\end{aligned}
$$

where

$$
r \equiv \frac{R_{c}}{R+R_{c}}
$$

The solution for $\Lambda(t)$ may be obtained, and then, for the period $T_{s}$ between $t=(k-2) T_{s}$ and $t=(k-1) T_{s}$, the voltseconds stored are given by

$$
\Lambda_{k-1}=\Lambda\left[t=(k-1) T_{s}\right]-\Lambda\left[t=(k-2) T_{s}\right]
$$

where $\Lambda\left[t=(k-2) T_{s}\right]=-\Lambda_{s}$, the negative saturation value from which $\Lambda$ starts at the beginning of each period $T_{s}$. It may be noted that if $R_{c} \rightarrow 0$, then $r \rightarrow 0$ and (25) reduces directly to (18), already obtained for the zero source resistance case.

After considerable algebriac manipulation, $\Lambda_{k-1}$ obtained from (25) and (27) may be expressed in the already mentioned form

$$
\Lambda_{k-1}=\Lambda_{0}+\lambda \sin \left[(k-1)\left(\frac{2 \pi \omega}{\omega_{s}}\right)-\theta-\phi_{m}\right]
$$

where

$$
\Lambda_{0}=\left(\frac{1-e^{-\alpha}}{\alpha}\right)\left[(1-r) V_{c}-r R\left(I_{0}-\frac{\Lambda_{s}}{L}\right)\right] T_{s}
$$




$$
\begin{gathered}
\lambda=\left(\frac{1-e^{-\alpha}}{\alpha}\right)(1-r) \nu_{c} T_{s} \\
\cdot \sqrt{\frac{1+e^{-\alpha}\left(\frac{\alpha}{1-e^{-\alpha}}\right)^{2}\left(\frac{2}{\alpha}\right)^{2} \sin ^{2}\left(\frac{\pi \omega}{\omega_{s}}\right)}{1+\left(\frac{2}{\alpha}\right)^{2}\left(\frac{\pi \omega}{\omega_{s}}\right)^{2}}} \\
\phi_{m}=\tan ^{-1}\left[\frac{\tan \left(\frac{\pi \omega}{\omega_{s}}\right)+\left(e^{\alpha}-1\right) \operatorname{cosec}\left(\frac{2 \pi \omega}{\omega_{s}}\right)-\left(\frac{\alpha \omega_{s}}{\pi \omega}\right)}{1+\left(\frac{\alpha \omega_{s}}{\pi \omega}\right)\left[\tan \left(\frac{\pi \omega}{\omega_{s}}\right)+\left(e^{\alpha}-1\right) \operatorname{cosec}\left(\frac{2 \pi \omega}{\omega_{s}}\right)\right.}\right]
\end{gathered}
$$

in which

$$
\alpha \equiv r \frac{T_{s}}{\tau}=\frac{R_{c}}{R+R_{c}} \frac{T_{s}}{\tau} .
$$

As a partial check, it may be seen that these rather formidable expressions reduce to those of (20) through (22) for $R_{c} \rightarrow 0$. The complexity of these expressions occurs because, in each period $T_{s}$, the stored volt-seconds start from $-\Lambda_{s}$, and $\Lambda_{k-1}$ is a transient solution of the differential equation(25). Also, neither the voltage nor current at the control winding is purely sinusoidal when $R_{c} \neq 0$, even though the control voltage is sinusoidal.

When $\Lambda_{k-1}$ given by (28), $D_{k}$ is given by (9) as

$$
D_{k}=D+d \sin \left[(k-1)\left(\frac{2 \pi \omega}{\omega_{s}}\right)-\theta-\phi_{m}\right]
$$

where

$$
D=\frac{\Lambda_{0}}{V_{g} T_{s}}
$$

is the dc duty ratio and

$$
d=\frac{\lambda}{V_{g} T_{s}}
$$

may be identified as the amplitude of the ac duty ratio. Note that $0<D<1$, and $d<D$. With $D$ given by (33), the complete waveform of the voltage $v(t)$ at the filter input, shown in Fig. 3(C), is known. It remains to find the dc and fundamental ac component at the frequency $\omega$, shown in Fig. 3(D), which may be done by expressing $v(t)$ as a Fourier series. The actual procedure depends upon the nature of the relationship between the switching frequency $f_{s}$ and the control frequency $f$. If

$$
\frac{f}{f_{s}}=\frac{\omega}{\omega_{s}}=\frac{N}{M}=P
$$

where $N$ and $M$ are positive integers, so that $P$ is a rational number including zero and the integers, then $f$ and $f_{s}$ are said to be commensurate. It follows, with $T_{s}=1 / f_{s}$ and $T=1 / f$, that

$$
M T_{s}=N T
$$

or $N$ periods of the control frequency contain $M$ periods of the switching frequency. That is, the waveform $v(t)$ in Fig. $3(\mathrm{C})$ is repetitive with period $M T_{s}=N T$, and this is the shortest interval over which $v(t)$ can be integrated to obtain its Fourier series expansion. Fig. 3 (and also Fig. 4) shows the waveform for the particular values $M=10, N=1$. If $f$ and $f_{s}$ are not commensurate, so that $P$ is an irrational number, the waveform $v(t)$ is not periodic, and recourse must be taken to a double integration to obtain the Fourier components. Only the commensurate case will be treated here, since this is simpler and the results are the same as for the incommensurate case [1].

A convenient form for the Fourier series expansion of the voltage $v(t)$ at the filter input is

$$
v(t)=K_{0}+\sum_{n=1}^{\infty}\left|K_{n}\right| \sin \left(n \omega t+\angle K_{n}\right)
$$

where

$$
\begin{aligned}
& K_{0}=\frac{1}{2 \pi N} \int_{0}^{2 \pi N} v(t) d(\omega t) \\
& K_{n}=\frac{j}{\pi N} \int_{0}^{2 \pi N} v(t) \exp (-j n \omega t) d(\omega t) .
\end{aligned}
$$

The integration interval $2 \pi N$ covers $N$ periods of $f$ and $M$ periods of $f_{s}$. Since only the dc and the $\omega$ component of $v(t)$ are required, as shown in Fig. 3(D), only the $n=1$ term in (38) is needed, and the result can be expressed as

$$
v(t)=V+v \sin \left(\omega t-\theta-\phi_{m}-\phi_{d}\right)
$$

where

$$
\begin{aligned}
V & =\frac{1}{2 \pi N} \int_{0}^{2 \pi N} v(t) d(\omega t) \\
v & =\left|K_{1}\right| \\
\theta+\phi_{m}+\phi_{d} & =-\not K_{1}
\end{aligned}
$$

in which

$$
K_{1}=\frac{j}{\pi N} \int_{0}^{2 \pi N} v(t) \exp (-j \omega t) d(\omega t)
$$

The form for $v(t)$ to be used in (42) and (45) is that shown in Fig. 3(C), and expressed by

$v(t)=V_{\ell}, \quad(k-1) T_{s}<t<(k-1) T_{s}+T_{k}$

$v(t)=0, \quad(k-1) T_{s}+T_{k}<t<k T_{s} \quad k=1,2, \cdots M$. 
Equation (42) then becomes

$$
V=\frac{V_{\ell}}{2 \pi N} \sum_{k=1}^{M} \int_{(k-1) \omega T_{s}}^{(k-1) \omega T_{s}+\omega T_{k}} d(\omega t)
$$

which leads simply to the dc value

$$
V=D V_{\ell} \text {. }
$$

Equation (45) becomes

$$
K_{1}=\frac{j V_{\ell}}{\pi N} \sum_{k=1}^{M} \int_{(k-1) \omega T_{s}}^{(k-1) \omega T_{s}+\omega T_{k}} \exp (-j \omega t) d(\omega t)
$$

which, with $D_{k}=T_{k} / T_{s}$, leads to

$$
\begin{aligned}
K_{1}=\frac{V_{\ell}}{\pi N} \sum_{k=1}^{M} & {\left[\exp \left[-j \omega T_{s}(k-1)\right]\right.} \\
& -\exp \left[-j \omega T_{s}(k-1+D)\right] \\
& \left.-\exp \left[-j \omega T_{s}\left(D_{k}-D\right)\right]\right] .
\end{aligned}
$$

Consider the last exponential in (50). With $D_{k}$ substituted from (33), this factor is

$$
\begin{aligned}
\exp \left[-j \omega T_{s}\left(D_{k}-D\right)\right]= & \exp \left(-j 2 \pi\left(\frac{\omega}{\omega_{s}}\right) d \sin [(k-1)\right. \\
& \left.\left.\left(\frac{2 \pi \omega}{\omega_{s}}\right)-\theta-\phi_{m}\right]\right)
\end{aligned}
$$

Since the amplitude $v$ of the $\omega$ component in the filter input voltage $v(t)$ is proportional to $K_{1}$, and since the ac duty ratio amplitude $d$ is proportional to the amplitude $v_{c}$ of the ac control signal, (51) shows that $v$ is a nonlinear function of $v_{c}$. Although the analysis can be continued in general (a Bessel function results [1]), a linearization restriction will be imposed here. The requirement is that the exponent of (51) should be sufficiently small that the exponential may be approximated by the first two terms of its series expansion:

$$
\begin{aligned}
\exp \left[-j \omega T_{s}\left(D_{k}-D\right)\right] \approx & 1-j\left[2 \pi\left(\frac{\omega}{\omega_{s}}\right) \mathrm{d}\right] \\
& \cdot \sin \left[(k-1)\left(\frac{2 \pi \omega}{\omega_{s}}\right)-\theta-\phi_{m}\right]
\end{aligned}
$$

This makes $v$ proportional to $v_{c}$, so that the control DF is linearized (independent of control-signal ac amplitude). The restriction required to validate the approximation of (52) is

$$
2 \pi\left(\frac{\omega}{\omega_{s}}\right) d<<1
$$

Since $d<D<1$, this restriction is not very severe for control frequency $\omega$ less than the switching frequency $\omega_{s}$. Further algebraic manipulation of (50), with the linearizing approximation of (52) included, eventually leads to

$$
\begin{aligned}
K_{1}= & {\left[\frac{V_{\ell}}{\left(\pi \omega / \omega_{s}\right)}\left(1-\exp \left[-j 2 D\left(\frac{\pi \omega}{\omega_{s}}\right)\right]\right)\right]_{\frac{\omega}{\omega_{s}}=1,2,3 \cdots} } \\
& +\left[d V_{\ell} \exp \left(-j\left[2 D\left(\frac{\pi \omega}{\omega_{s}}\right)+\theta+\phi_{m}\right]\right)\right]_{\frac{\omega}{\omega_{s}} \neq \frac{1}{2}, 1, \frac{3}{2}, \cdots} \\
& +\left[d V_{\ell} \exp \left(-j\left[2 D\left(\frac{\pi \omega}{\omega_{s}}\right)+\theta+\phi_{m}\right]\right)\right.
\end{aligned}
$$

$$
\left.\cdot\left(1-\exp \left[2 j\left(\theta+\phi_{m}\right)\right]\right)\right]_{\frac{\omega}{\omega_{s}}=\frac{1}{2}, 1, \frac{3}{2}, \cdots} .
$$

The significance of the contributions to this result is as follows. The term in the first square bracket is independent of $d$, so it exists in the absence of an ac control signal; it represents the switching frequency and its harmonics. The term in the second square bracket is proportional to $d$, and represents the $\omega$ component that results directly from the ac control signal. The term in the third square bracket, also proportional to $d$, represents the combination of the $\omega \mathrm{com}$ ponent that results directly from the ac control signal and the appropriate sideband of the switching frequency. For example, for $\omega / \omega_{s}=1 / 2$, the combination is of the direct component $\omega=(1 / 2) \omega_{s}$ and the sideband $\omega_{s}-\omega=$ $(1 / 2) \omega_{s}$. It is seen that the amplitude of the $\omega$ component for $\omega / \omega_{s}=1 / 2,1,3 / 2, \cdots$ can be as great as twice the amplitude for neighboring frequencies, the actual amplitude depending upon the phase $\theta$ of the control signal with respect to the switching frequency.

Since the components of $K_{1}$ for $\omega / \omega_{s}=1,2,3, \cdots$ are present even in the absence of an ac control signal, the term in the first square bracket in (54) is excluded from the DF representation. The remaining two terms may be combined into a single term in the following manner:

$$
\begin{aligned}
K_{1}= & d V_{\ell} \exp \left(-j\left[2 \pi\left(\frac{\pi \omega}{\omega_{s}}\right)+\theta+\phi_{m}\right]\right) \\
& \cdot\left(1-k \exp \left[2 j\left(\theta+\phi_{m}\right)\right]\right)
\end{aligned}
$$

where $k=1$ for the "special-case" frequencies $\omega / \omega_{s}=1 / 2$, $1,3 / 2, \cdots$, and $k=0$ otherwise. The magnitude and phase of the $\omega$ component of the filter input voltage $v(t)$ are then obtained from (43), (44), and (55) as

$$
\begin{aligned}
v & =d V_{\ell}\left|1-k \exp \left[2 j\left(\theta+\phi_{m}\right)\right]\right| \\
\phi_{d} & =2 D\left(\frac{\pi \omega}{\omega_{s}}\right)-\angle 1-k \exp \left[2 j\left(\theta+\phi_{m}\right)\right] .
\end{aligned}
$$


The results may now be assembled as follows. The dc control transfer function is obtained from (48), (34), and (29) as

$$
V=\frac{V_{\ell}}{V_{g}}\left(\frac{1-e^{-\alpha}}{\alpha}\right)\left[(1-r) V_{c}-r R\left(I_{0}-\frac{\Lambda_{s}}{L}\right)\right]
$$

where

$$
\begin{aligned}
r & =\frac{R_{c}}{R+R_{c}} \\
\alpha & =\frac{T_{s}}{\tau}=\frac{R_{c}}{R+R_{c}} \frac{T_{s}}{\tau}
\end{aligned}
$$

It is convenient to normalize the ac control DF $F_{c}^{\prime}(j \omega)$ to its zero-frequency value $F_{c}^{\prime}(j 0)$, and to define the resulting normalized control signal to filter input voltage DF and $H_{c}^{\prime}$, which is obtained from (12), with insertion of (56), (57), (35), and (30), as

$$
\begin{aligned}
H_{c}^{\prime} \equiv \frac{F_{c}^{\prime}(j \omega)}{F_{c}^{\prime}(j 0)}= & A \exp \left[-j\left[2 D\left(\frac{\pi \omega}{\omega_{s}}\right)+\phi_{m}\right]\right] \\
& \cdot\left(1-k \exp \left[2 j\left(\theta+\phi_{m}\right)\right]\right)
\end{aligned}
$$

where

$$
F_{c}^{\prime}(j 0)=\frac{V_{\ell}}{V_{g}}\left(\frac{1-e^{-\alpha}}{\alpha}\right) \frac{R}{R+R_{c}}
$$

and

$$
\begin{gathered}
A \equiv \sqrt{\frac{1+e^{-\alpha}\left(\frac{\alpha}{1-e^{-\alpha}}\right)^{2}\left(\frac{2}{\alpha}\right)^{2} \sin ^{2}\left(\frac{\pi \omega}{\omega_{s}}\right)}{1+\left(\frac{2}{\alpha}\right)^{2}\left(\frac{\pi \omega}{\omega_{s}}\right)^{2}}} \\
\phi_{m}=\tan ^{-1}\left[\frac{\tan \left(\frac{\pi \omega}{\omega_{s}}\right)+\left(e^{\alpha}-1\right) \operatorname{cosec}\left(\frac{2 \pi \omega}{\omega_{s}}\right)-\left(\frac{\alpha \omega_{s}}{\pi \omega}\right)}{1+\left(\frac{\alpha \omega_{s}}{\pi \omega}\right)\left(\tan \left(\frac{\pi \omega}{\omega_{s}}\right)+\left(e^{\alpha}-1\right) \operatorname{cosec}\left(\frac{2 \pi \omega}{\omega_{s}}\right)\right)}\right]
\end{gathered}
$$

with

$$
k= \begin{cases}0, & \omega / \omega_{s} \neq 1 / 2,1,3 / 2, \cdots \\ 1, & \omega / \omega_{s}=1 / 2,1,3 / 2, \cdots\end{cases}
$$

Equation (61) shows that a polar plot of the ac control DF $H_{c}^{\prime}$ is a trajectory traced out by a phasor of length $A$ and angle $-\left[2 D\left(\pi \omega / \omega_{s}\right)+\phi_{m}\right]$, but that at the special-case frequencies $\omega / \omega_{s}=1 / 2,1,3 / 2, \cdots(k=1)$, the total phasor is the sum of the first phasor with $k=0$ and a second phasor of equal magnitude, but with an angle that depends upon the phase $\theta$ of the control ac signal with respect to the switching frequency. The second phasor describes a circle, centered on the end of the first phasor, as $\theta+\phi_{m}$ varies from 0 to $\pi$. A qualitative sketch of such a polar plot of $H_{c}^{\prime}$ is shown in Fig. 6. At the frequencies $\omega / \omega_{s}=1 / 2,1,3 / 2, \cdots$,

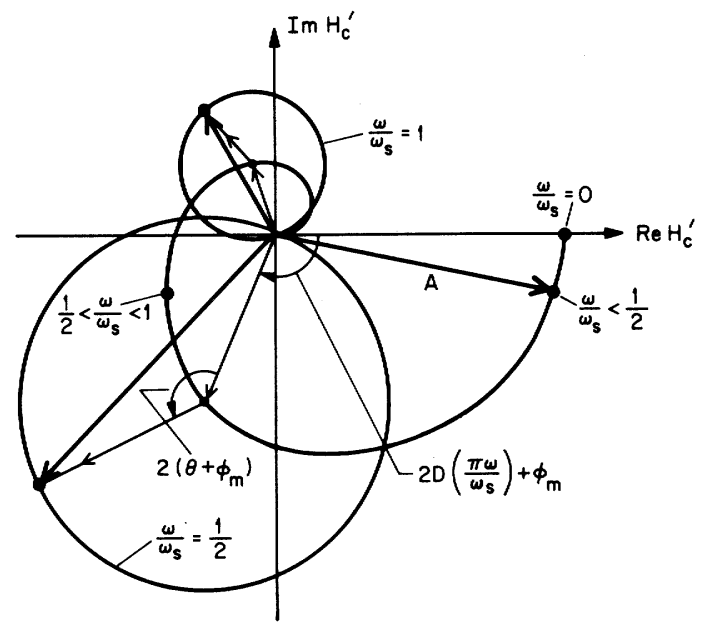

Fig. 6. Polar plot of the normalized control DF $H_{c}^{\prime}$, showing the two phasor components at $\omega / \omega_{s}=1 / 2,1$, $3 / 2 \cdots$, and the single phasor component at other frequencies.

the total phasor has an amplitude that varies from zero to $2 A$, and a phase angle that varies up to $\pm \pi / 2$ from $-\left[2 D\left(\pi \omega / \omega_{s}\right)+\phi_{m}\right]$, depending upon $\theta$.

Separate magnitude and phase plots of the ac control DF $H_{c}^{\prime}$ versus frequency are shown in Figs. 7 and 8, with $\alpha$ as a parameter. From (61),

$$
\begin{aligned}
\left|H_{c}^{\prime}\right|\left[\frac{\omega}{\omega_{s}} \neq 1 / 2,1,3 / 2, \cdots\right]= & A \\
\left\langle H_{c}^{\prime}\left[\frac{\omega}{\omega_{s}} \neq 1 / 2,1,3 / 2, \cdots\right]=\right. & -\left[2 D\left(\frac{\pi \omega}{\omega_{s}}\right)+\phi_{m}\right] \\
\left|H_{c}^{\prime}\right|\left[\frac{\omega}{\omega_{s}}=1 / 2,1,3 / 2, \cdots\right]= & 2 A \sin \left(\theta+\phi_{m}\right) \\
\left\langle H_{c}^{\prime}\left[\frac{\omega}{\omega_{s}}=1 / 2,1,3 / 2, \cdots\right]=\right. & -\left[2 D\left(\frac{\pi \omega}{\omega_{s}}\right)+\phi_{m}\right] \\
& -\left[\left(\theta+\phi_{m}\right)-\frac{\pi}{2}\right] .
\end{aligned}
$$

For $\alpha=0$, that is, for a voltage-driven control signal with $R_{c}=0$, the magnitude and phase factors $A$ and $\phi_{m}$ due to the magnetic modulator reduce to

$$
\begin{aligned}
A & =\frac{\sin \left(\pi \omega / \omega_{s}\right)}{\left(\pi \omega / \omega_{s}\right)} \\
\phi_{m} & =\frac{\pi \omega}{\omega_{s}}
\end{aligned}
$$

In Fig. 7, magnitude plots are given for $\alpha=0,1.5$, and 10 . The frequency dependence stems entirely from the modulator. In Fig. 8, phase plots are given for $\alpha=0$ and 1.5, with the dc duty ratio $D=0.5$. The total phase lag is made up of the two components shown, $2 D\left(\pi \omega / \omega_{s}\right)$ due to the power stage and $\phi_{m}$ due to the modulator.

The zero to double-amplitude possibility expressed by (68) is represented in Fig. 7 by "spikes" superimposed upon 


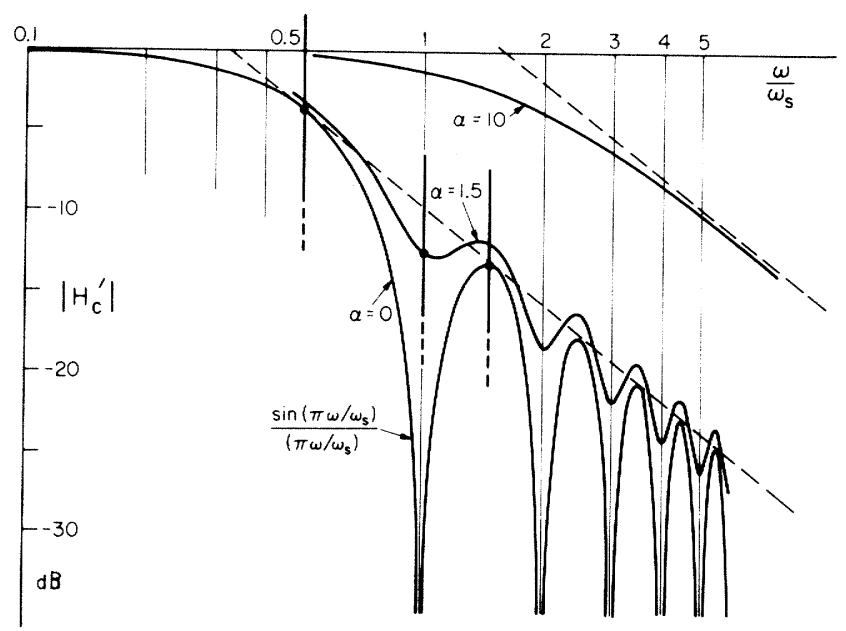

Fig. 7. Theoretical magnitude versus frequency plot of the control DF $H_{c}^{\prime}$, for $\alpha=0,1.5$, and 10 , showing the "spikes" at $\omega / \omega_{c}=1 / 2$, $1,3 / 2 \cdots$

the amplitude $A$ at the special-case frequencies $\omega / \omega_{s}=1 / 2$, $1,3 / 2, \cdots$. The spike extends $6 \mathrm{~dB}$ above and $\infty \mathrm{dB}$ below the corresponding amplitude $A$. To avoid cluttering the picture, only a few of these spikes are shown in Fig. 7, but they are present at $\omega / \omega_{s}=1 / 2,1,3 / 2, \cdots$ for all values of $\alpha$. The $\pm \pi / 2$ additional phase lag expressed by (69) is similarly represented in Fig. 8 by $\pm \pi / 2$ spikes superimposed upon the phase lag $\left[2 D\left(\pi \omega / \omega_{s}\right)+\phi_{m}\right]$ at the special-case frequencies $\omega / \omega_{s}=1 / 2,1,3 / 2, \cdots$. [The $\pm \pi / 2$ range is given by (69) for $0<\theta+\phi_{m}<\pi$; for $\pi<\left(\theta+\phi_{m}\right)<2 \pi$, the additional lag $\pi$ is cancelled by the sign reversal of the amplitude factor given by (68).] Again, only a few of the spikes are shown in Fig. 8.

The most important feature of the results displayed in the control-signal DF plots of Figs. 7 and 8 is that the performance of the modulator and power-stage subsystem is improved if the modulator is driven from a current source rather than from a voltage source. The performance is improved in the sense that the frequency response of the control DF is extended; the magnitude decay and the phase lag at a given frequency are both less for $\alpha>0$ than for $\alpha=0$. Extended frequency response translates directly into improved gain and phase margins when the subsystem is inside a feedback loop. The improvement is quite substantial; at $\omega / \omega_{s}=0.8$, for example, the phase lag for $\alpha=1.5$ is less by about $\pi / 2$ than that for $\alpha=0$, but the magnitude increase is only about $3.5 \mathrm{~dB}$. The degree of improvement in the frequency response is, however, limited by the maximum value of $\alpha$, given by (60), with $R_{c} \rightarrow \infty$ for a currentsource control signal:

$$
\alpha_{\max }=\frac{T_{s}}{\tau} .
$$

\section{Experimental Results}

Experimental results for the control-signal DF $H_{c}^{\prime}$ were obtained on a circuit embodying the principle illustrated in

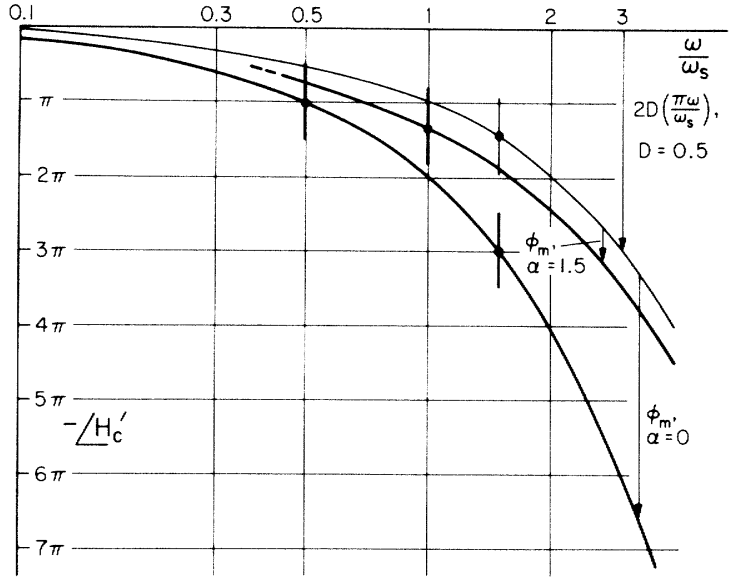

Fig. 8. Theoretical phase versus frequency plot of the control DF $H_{c}^{\prime}$, showing the component $2 D\left(\pi \omega / \omega_{s}\right)$ from the power stage for $D=0.5$, and the component $\phi_{m}$ from the modulator for $\alpha=0$ and 1.5 .

Fig. 2. Each core was a Magnetics Inc. 51056-2H, Type 48 alloy. The control and gate windings each consisted of 500 turns of no. 26 wire. The clock square-wave was obtained from an Exact 301 function generator at $1 \mathrm{kHz}$, so the switching frequency was $f_{s}=2 \mathrm{kHz}$ with $T_{s}=0.5 \mathrm{~ms}$. The peak value of the gate square wave was $V_{g}=14$ volts. Since the modulated square-wave voltage $v(t)$ at the filter input is the same as that at the modulator output (except for a scale factor and phase reversal), the power stage was omitted and replaced by a Schmitt trigger to sharpen the modulator output waveform, which is soft because of the soft saturation characteristic of the cores. The control dc and ac signals were obtained from a low-output impedance amplifier, either directly for voltage drive $V_{c}\left(R_{c}=0\right)$, or via a common-emitter transistor stage for current drive $I_{c}=V_{c} / R_{c}\left(R_{c}=\infty\right)$. A Hewlett-Packard 302A wave analyzer was used to measure the magnitude of the control-signal frequency component in the Schmitt trigger modulated square-wave output waveform $v(t)$. In the BFO mode, the wave analyzer provides a signal at the frequency to which the voltmeter is tuned, so this signal was used to supply the control-signal ac component.

The experimental results for $\left|H_{c}^{\prime}\right|$ obtained in this way are shown in Fig. 9. The solid line is the theoretical result for $\alpha=0$ taken from Fig. 7, and it is seen that the corresponding experimental points for voltage drive $\left(R_{c}=0, \alpha=0\right)$ agree quite closely with the theoretical curve. The experimental points for current drive $\left(R_{c}=\infty, \alpha=\alpha_{\max }\right)$ also lie on a curve of the expected shape, and are well fitted by the theoretical curve for $\alpha=0.95$. Hence, from (72),

$$
\tau=\frac{T_{s}}{\alpha_{\max }}=\frac{0.5}{0.95}=0.53 \mathrm{~ms} .
$$

To verify the presence of the "spikes" in the $\left|H_{c}^{\prime}\right|$ plot, the ac control signal must be synchronized with the switching frequency. In the experimental setup described, this 


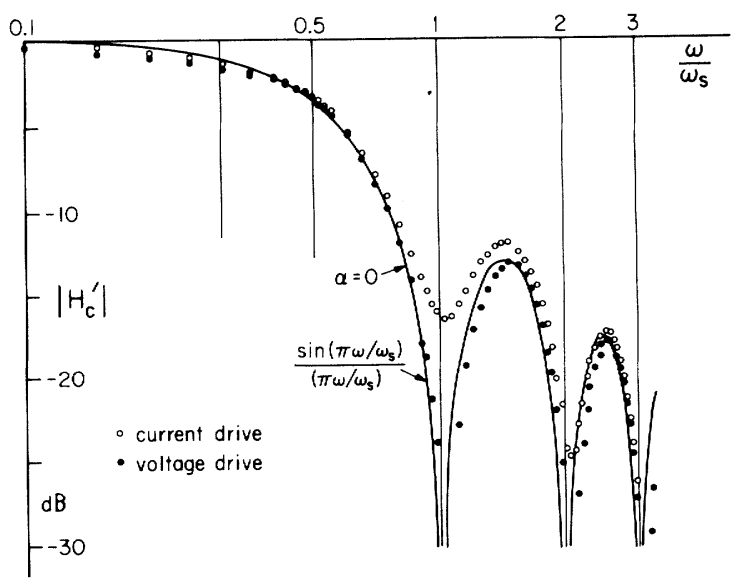

Fig. 9. Experimental magnitude versus frequency plot of $H_{c}^{\prime}$ for voltage drive and for current drive.

can be done very simply for $\omega / \omega_{s}=1 / 2$ by use of the sinewave output of the Exact function generator, which is available simultaneously with the square-wave output at the same frequency used for the clock, which is $\omega_{s} / 2$. The sine-wave output at $\omega=\omega_{s} / 2$ was applied as the ac control signal through a phase-shifting network, and the amplitude of the control signal was adjusted, for each value of phase angle, to the (constant) value previously used for the measurements at other frequencies. Measurements were made for voltage drive, and are shown in Fig. 10. The results agree well with the theoretical curve, also shown, expressed by (68), with $A$ and $\phi_{m}$ given by (70) and (71) for $\omega / \omega_{s}=1 / 2$ :

$$
\left|H_{c}^{\prime}\right|\left[\frac{\omega}{\omega_{s}}=\frac{1}{2}\right]=\frac{2}{\pi / 2} \sin \left(\theta+\frac{\pi}{2}\right)
$$

The measurements shown in Figs. 9 and 10 were all taken at a dc duty ratio $D=0.9$. Since the $302 \mathrm{~A}$ wave analyzer makes only magnitude measurements, experimental verification of the theoretical phase relations of Fig. 8 was not obtained. However, the agreement between the theoretical and the experimental magnitude relations is sufficiently good to inspire confidence in the theoretical expression for the control signal DF.

Experimental measurements were also made of the dc control transfer characteristic. From (34) and (29), the theoretical relation between the dc duty ratio $D$ and the control signal is

$$
D=\frac{1}{V_{g}}\left(\frac{1-e^{-\alpha}}{\alpha}\right)\left[(1-r) V_{c}-r R\left(I_{0}-\frac{\Lambda_{s}}{L}\right)\right] .
$$

For voltage drive $V_{c}$ with $R_{c}=0, r=0$ and $\alpha=0$, so that

$$
\left.D\right|_{\alpha=0}=\frac{V_{c}}{V_{g}} \text {. }
$$

For current drive $I_{c}=V_{c} / R_{c}$ with $R_{c}=\infty, r=1$ and $\alpha=\alpha_{m}$,

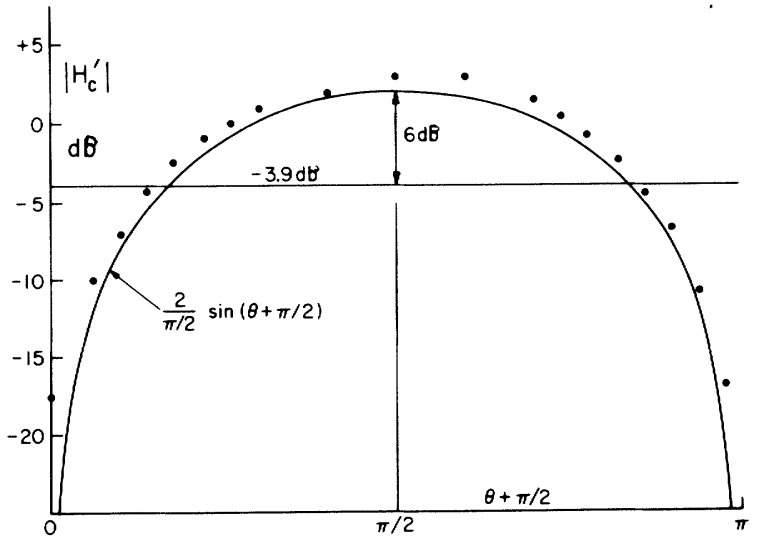

Fig. 10. Theoretical and experimental magnitude of $H_{C}^{\prime}$ versus the phase $\theta$ of the control signal, for $\omega / \omega_{s}=1 / 2$.

so that

$$
\left.D\right|_{\alpha=\alpha_{m}}=\left(\frac{1-e^{-\alpha_{m}}}{\alpha_{m}}\right) \frac{R}{V_{g}}\left[I_{c}-\left(I_{0}-\frac{\Lambda_{s}}{L}\right)\right] .
$$

Experimental measurements of $\left.D\right|_{\alpha=0}$ versus $V_{c}$ and $\left.D\right|_{\alpha=\alpha_{m}}$ versus $I_{c}$ are shown in Figs. 11 and 12. The duty ratio was determined by direct observation of the Schmitt trigger output waveform $v(t)$ on an oscilloscope. The $\left.D\right|_{\alpha=0}$ transfer characteristic is linear over essentially the entire range $0<D<1$, but the $\left.D\right|_{\alpha=\alpha_{m}}$ characteristic becomes nonlinear at both ends of the range. This is because the actual cores do not have the sharp saturation characteristic assumed in the model of Fig. 5. However, a substantial part of the range is linear with a slope of $1 / 0.54 \mathrm{~mA}$. The measured slope of the $\left.D\right|_{\alpha=0}$ characteristic is $1 / 12$ volt, and from (76) and (77), the value of $R$ can be obtained from

$$
\left(\frac{1-e^{-\alpha} m}{\alpha_{m}}\right) R=\frac{12 \mathrm{~V}}{0.54 \mathrm{~mA}}=22.2 \mathrm{~K}
$$

With the previously determined value $\alpha_{m}=0.95$, the result is $R=34 k$.

As described above, the performance of the cores in the modulator permits experimental determination of the core second-order parameters $R$ and $\tau$. However, the first-order core parameters $\Lambda_{s}$ and $I_{0}$ can also be obtained. An independent measurement showed that the total volt-seconds supportable by the core with 500 turns was $2 \Lambda_{s}=3.9 \mathrm{~V} \cdot \mathrm{ms}$, so that $\Lambda_{s}=1.95 \mathrm{~V} \cdot \mathrm{ms}$. This converts to $B_{s}=9.1 \mathrm{kG}$, compared with the manufacturer's nominal value of $11.6 \mathrm{kG}$. The value of $I_{0}$ can be obtained from the intercept of the $\left.D\right|_{\alpha=\alpha_{m}}$ characteristic with the current axis, from Fig. 12 and (77), as

$$
I_{0}-\frac{\Lambda_{s}}{L}=0.72 \mathrm{~mA}
$$

The nonsaturated inductance $L=\pi R=0.53 \mathrm{~ms} \times 34 k=$ $18 \mathrm{H}$, so $\Lambda_{s} / L=1.95 / 18=0.11 \mathrm{~mA}$ and $I_{0}=0.72+0.11$ 


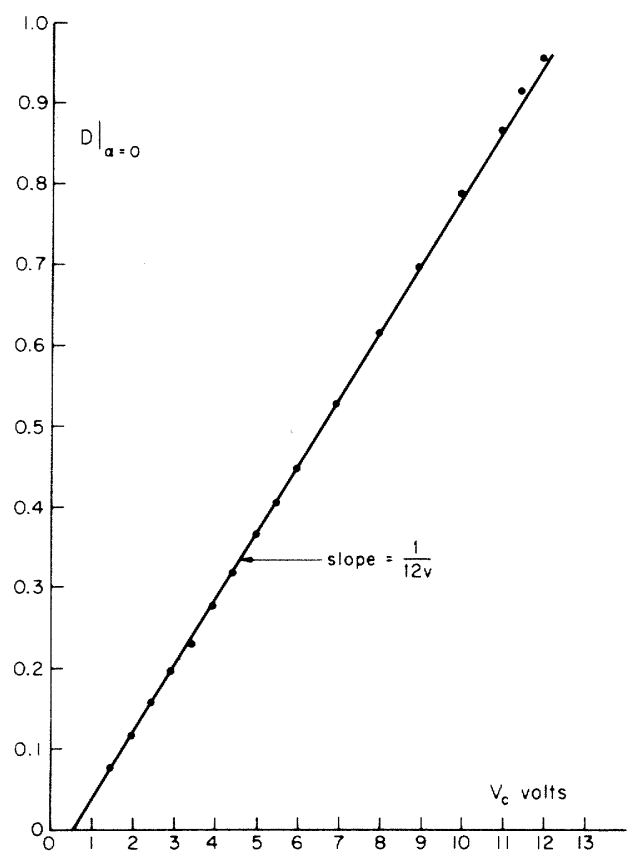

Fig. 11. Duty ratio $D$ versus control voltage $V_{c}$ for voltage drive $(\alpha=0)$.

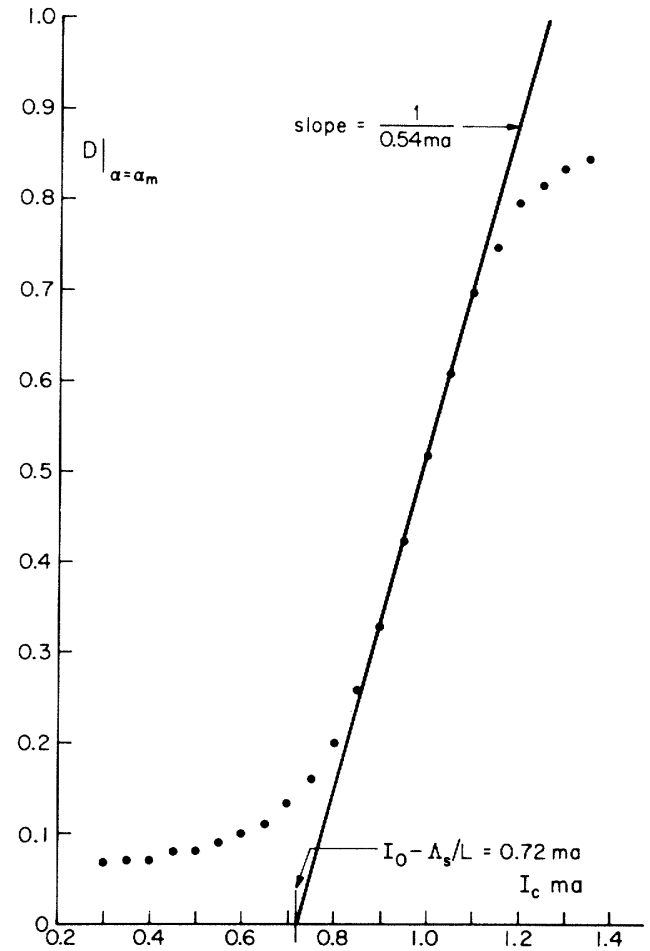

Fig. 12. Duty ratio $D$ versus control current $I_{C}$ for current drive $\left(\alpha=\alpha_{m}\right)$.
$=0.83 \mathrm{~mA}$. This converts to $H_{0}=0.12$ oersted, compared with the manufacturer's nominal value of 0.1 oersted.

It may be observed that the measured slope of the $\left.D\right|_{\alpha=0}$ characteristic in Fig. 11, 1/12 volt, does not agree with the value of $1 / V_{g}$ predicted by (76) when $V_{g}=14$ volts. This is because the voltage that actually appears across the gate winding is less than $V_{g}$ by a diode drop of about 0.6 volt, and by the drop in the load resistance $R_{g}$ due to the core magnetizing current. Although the magnetizing current changes during the interval $T_{s}$ that the core is resetting (because of the finite inductance $L$ ), an approximate average value is $I_{m}=I_{0}+(1 / R) d \Lambda / d t$. If the gate winding voltage is assumed to be the measured value $d \Lambda / d t=12$ volts, $I_{m}=$ $0.83+12 / 34=1.18 \mathrm{~mA}$. The value of $R_{g}$ used in the experimental setup was $R_{g}=1.35 \mathrm{k}$, so the effective gate winding voltage is $14-0.6-(1.18 \times 1.35)=14-0.6-1.6$ $=11.8$ volts, in satisfactory agreement with the observed 12 volts. This effective value of $V_{g}$ should, of course, also be employed in the $\left.D\right|_{\alpha=\alpha_{m}}$ characteristic of (77). The discrepancy in the offset of the $\left.D\right|_{\alpha=0}$ characteristic observed in Fig. 11 occurs because the effective control winding voltage is less than $V_{c}$ by a diode drop of about 0.6 volt.

\section{The Line Describing Function}

As for the control DF, the analytic derivation of the line DF is in two parts. In the first part an expression for the $k$ th duty ratio $D_{k}$ is obtained, and in the second part the coefficient $K_{1}$ of the $\omega$ component in the filter input voltage waveform $v(t)$ is obtained by Fourier analysis.
A qualitative description of the signal conditions has been given in Section II, with reference to Fig. 4. The line voltage has a dc plus a sinusoidal component, given by (13), and the gate voltage is proportional to the line voltage, as in (14). The control signal is constant, so the volt-seconds stored in each interval $T_{s}$ are constant at $\Lambda_{0}$. These voltseconds are recovered under the action of the gate voltage $v_{g}(t)$ to determine $T_{k}$ by

$$
\int_{(k-1) T_{s}}^{(k-1) T_{s}+T_{k}} v_{g}(t) d t=\Lambda_{0} .
$$

The duty ratio $D_{k}$ is then given by

$$
\begin{aligned}
B \int_{(k-1)}^{(k-1)+D_{k}}\left[V_{\ell}+v_{\ell} \sin (\omega t-\theta)\right] d\left(\frac{t}{T_{s}}\right) & =\Lambda_{0} \\
& =D B V_{\ell}
\end{aligned}
$$

where the second equality is obtained by recognition that $D_{k}=D$, the dc duty-ratio, when $v_{\ell}=0$. Evaluation of the integral in (81) leads to a transcendental relation for $D_{k}$. However, a closed-form solution can be obtained as

$$
D_{k}=D+d \sin \left[(k-1)\left(\frac{2 \pi \omega}{\omega_{s}}\right)-\theta+\frac{D \pi \omega}{\omega_{s}}\right]
$$


where

$$
\begin{aligned}
d \equiv & -D \frac{\sin \left(D \pi \omega / \omega_{s}\right)}{\left(D \pi \omega / \omega_{s}\right)} \\
& \cdot \frac{v_{\ell} / V_{\ell}}{1+\left(v_{\ell} / V_{\ell}\right) \sin \left[(k-1)\left(2 \pi \omega / \omega_{s}\right)-\theta+D \pi \omega / \omega_{s}\right]}
\end{aligned}
$$

under the restriction

$$
2 \pi\left(\frac{\omega}{\omega_{s}}\right) d<<1
$$

Equation (82) corresponds to (33), obtained for controlsignal variations. Also, the restriction of (84) is the same as that required in the control-signal analysis, (53). It may be noted that the restriction does not involve $v_{\ell} / V_{\ell}$, and so (82) is valid even for large fractional line voltage variations. However, $d$ is independent of $k$, and so the $D_{k}$ points lie on a sine wave only if $v_{\ell} / V_{\ell}<<1$.

The expression for the Fourier coefficient $K_{1}$ of the $\omega$ component in the filter input waveform $v(t)$ is established in the same way as for control-signal variations in (38) through (49), the only difference being that $v(t)=V_{Q}+v_{\ell}$ $\sin (\omega t-\theta)$ instead of $V_{Q}$ during the switch on-times $T_{k}$. Hence, for line variations, the equation for $K_{1}$ corresponding to (49) is

$$
\begin{aligned}
K_{1}= & \omega T_{s} \frac{j V_{\ell}}{\pi N} \sum_{k=1}^{M} \int_{(k-1)}^{(k-1)+D_{k}}\left[1+\left(\frac{v_{\ell}}{V_{\ell}}\right) \sin (\omega t-\theta)\right] \\
& \cdot \exp (-j \omega t) d\left(\frac{t}{T_{s}}\right)
\end{aligned}
$$

Again, commensurate frequencies are assumed such that $\omega / \omega_{s}=N / M$, where $N$ and $M$ are integers. Evaluation of the integral subject to the restriction of (84) permits $K_{1}$ to be expressed as a linear function of $D_{k}$, which can then be substituted by (82). Evaluation of the $k$ summations then leads to three groups of terms, as in the control variations case. The harmonics of the switching frequency, present even in the absence of line variations, are irrelevant, and the sideband combinations can be neglected, since it is unlikely that line variations synchronous with the switching frequency would exist. The remaining term that results directly from the ac line component is

$$
K_{1}=D v_{\ell} e^{j \theta}\left(1-\frac{1-\exp \left[-j 2 D\left(\pi \omega / \omega_{s}\right)\right]}{j 2 D\left(\pi \omega / \omega_{s}\right)}\right) \text {. }
$$

The ac line DF is then $F_{\ell}^{\prime}(j \omega)=K_{1} /\left(v_{\ell} e^{-j \theta}\right)$, and it is convenient to normalize $F_{\ell}^{\prime}(j \omega)$ to its infinite-frequency value $F^{\prime}(j \infty)$, and to define the resulting normalized line to filter input voltage DF as $H_{\ell}^{\prime}$, which is

$$
H_{\ell}^{\prime} \equiv \frac{F_{\ell}^{\prime}(j \omega)}{F_{\ell}^{\prime}(j \infty)}=1-\frac{1-\exp \left[-j 2 D\left(\pi \omega / \omega_{s}\right)\right]}{j 2 D\left(\pi \omega / \omega_{s}\right)}
$$

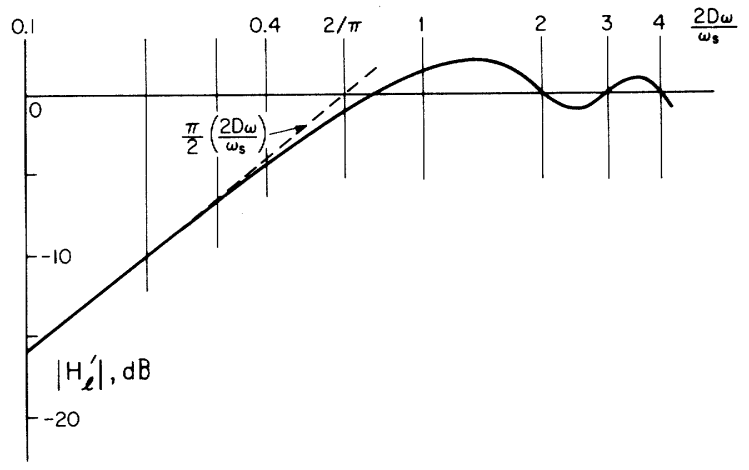

Fig. 13. Theoretical magnitude versus frequency plot of the line DF $H_{l}^{\prime}$.

where

$$
F_{\ell}^{\prime}(j \infty)=D
$$

The magnitude and phase of the ac line DF $H_{\ell}^{\prime}$ are given by

$$
\left|H_{\ell}^{\prime}\right|=\sqrt{\left(1-\frac{\sin \left(2 D \pi \omega / \omega_{s}\right)}{2 D \pi \omega / \omega_{s}}\right)^{2}+\left(\frac{\sin ^{2}\left(D \pi \omega / \omega_{s}\right)}{D \pi \omega / \omega_{s}}\right)^{2}}
$$

$L_{\ell}^{\prime}=\tan ^{-1}\left(\frac{2 \sin ^{2}\left(D \pi \omega / \omega_{s}\right)}{\left(2 D \pi \omega / \omega_{s}\right)-\sin \left(2 D \pi \omega / \omega_{s}\right)}\right)$.

Since the dc duty ratio $D$ always appears as a multiplier of $\omega$, a single plot of $\left|H_{\ell}^{\prime}\right|$ may be made as a function of $2 D \omega / \omega_{s}$, as shown in Fig. 13. As anticipated, $\left|H_{\ell}^{\prime}\right|$ is zero at zero frequency, when the line change applied to the power switch is exactly compensated by the change of duty ratio resulting from the corresponding change in gate voltage. However, as the frequency of the line variations increases, the increasing phase delay through the modulator prevents complete compensation; ultimately, for frequencies such that $2 D \omega / \omega_{s}$ is greater than about $2 \pi$, there is essentially no compensation and the line variations appear unmodified in the filter input voltage. Since in a practical application the phase $\angle H_{\ell}^{\prime}$ is of little concern, a plot of $\angle H_{\ell}^{\prime}$ is not given; however, it can be seen from (90) that $\left\langle H_{\ell}^{\prime}=k / 2\right.$ at zero frequency, and, ultimately, for high frequencies, decreases to zero.

For frequencies sufficiently low that $\left(2 D \omega / \omega_{s}\right)<<2 / \pi$, (89) and (90) reduce to

$$
\begin{aligned}
& \left|H_{\ell}^{\prime}\right|=\frac{\pi}{2}\left(\frac{2 D \omega}{\omega_{s}}\right) \\
& L_{\ell}^{\prime}=\frac{\pi}{2}-\tan ^{-1}\left(\frac{\pi}{3} \frac{2 D \omega}{\omega_{s}}\right) .
\end{aligned}
$$

\section{Conclusions}

A particular pulsewidth modulator and power-stage subsystem that has been widely used in practical switchingmode dc regulators has been analyzed. The subsystem may 
be classed as a buck power stage driven by a clocked, uniformly sampling, integrating modulator employing squareloop cores, whose principle of operation is illustrated in Fig. 2 .

The analysis leads to an expression for the describing function $F_{c}^{\prime}(j \omega)$ that relates a sinusoidal control signal at frequency $\omega$ at the modulator input to the resulting source frequency component of the filter input voltage. The overall describing function from the control signal to the output voltage is then $F_{c}(j \omega)=F_{c}^{\prime}(j \omega) F(j \omega)$, where $F(j \omega)$ is the filter linear transfer function. When the control input is voltage driven (zero source resistance), normal operation is independent of core parameters, and the normalized control DF $H_{c}^{\prime} \equiv F_{c}^{\prime}(j \omega) / F_{c}^{\prime}(j 0)$ is of the form $\sin \left(\pi \omega / \omega_{s}\right) /\left(\pi \omega / \omega_{s}\right)$. However, the frequency response is extended when the control signal is driven from a nonzero resistance source, as expressed by (61) and shown in Figs. 7 and 8. Extension of the frequency response is quantitatively related to two second-order core parameters, the "loop widening" resistance $R$ and the nonsaturated inductance $L$. The theoretical results are confirmed in Fig. 9 by experimental measurements made on a practical circuit. The complete knowledge of the control DF thus obtained is valuable in optimizing the design of feedback regulators in which this particular subsystem is used.

Analysis is also presented that leads to an expression for the describing function $F_{\ell}^{\prime}(j \omega)$ that relates a sinusoidal line voltage variation at frequency $\omega$ to the resulting source frequency component of the filter input voltage. The overall describing function from the line voltage to the output voltage is then $F_{\ell}(j \omega)=F_{\ell}^{\prime}(j \omega) / F(j \omega)$. When the modulator gate voltage is made proportional to the line voltage, the normalized line DF $H_{\ell}^{\prime} \equiv F_{\ell}^{\prime}(j \omega) / F^{\prime}(j \omega)$ is zero for zerofrequency variations, but increases with frequency, as shown by (87) and in Fig. 13. Thus, even though complete cancellation of line variations can be obtained at zero frequency, line "feedthrough" occurs at higher frequencies, and the analytical results derived here can be used to determine the overall line sensitivity of the output voltage in a complete feedback regulator system.

\section{Reference}

[1] G.W. Wester and R.D. Middlebrook, "Low-frequency characterization of switched dc-dc converters," IEEE Trans. Aerospace and Electronic Systems, this issue. pp. 376-385.

R.D. Middlebrook (S'55-M'56-SM'58), for a photograph and biography, please see this issue, p. 385. 